\title{
NONIMMERSIONS AND NONEMBEDDINGS OF QUATERNIONIC SPHERICAL SPACE FORMS
}

BY

TEIICHI KOBAYASHI

\begin{abstract}
We determine the orders of the canonical elements in $\mathrm{KO}$-rings of quaternionic spherical space forms $S^{4 n+3} / Q_{k}$ and apply them to prove the nonexistence theorems of immersions and embeddings of $S^{4 n+3} / Q_{k}$ in Euclidean spaces.
\end{abstract}

1. Statements of results. Let $Q_{k}=\left[x, y: x^{2^{k} \cdot 2}=y^{2}, x y x=y\right]$ be the generalized quaternion group of order $2^{k}(k>2)$. (Note that the relation $x^{2^{k-1}}=1$ follows from the above two relations.) Let $d_{1}: Q_{k} \rightarrow S^{3}=\mathrm{Sp}(1)=\mathrm{SU}(2)$ be the natural inclusion defined by $d_{1}(x)=\exp \left(2 \pi i / 2^{k-1}\right), d_{1}(y)=j$. Then $Q_{k}$ acts freely on the unit sphere $S^{4 n+3}$ in the quaternion $(n+1)$-space $H^{n+1}$ by the diagonal action $(n+1) d_{1}$ : $Q_{k} \rightarrow \operatorname{Sp}(n+1)$. The quotient manifold $S^{4 n+3} / Q_{k}$ is called the quaternionic spherical space form. D. Pitt [8] studied the structure of $K$ - and $K O$-rings of $S^{4 n+3} / Q_{k}$ and considered the problem of immersing or embedding $S^{4 n+3} / Q_{k}$ in Euclidean space $R^{m}$ using the techniques of M. F. Atiyah [1] (cf. also [5, Chapter 6] and [6, Chapter 3]).

The purpose of this note is to determine the orders of the canonical elements in $\widetilde{K O}\left(S^{4 n+3} / Q_{k}\right)$ and apply them to improve the nonexistence theorems of immersions and embeddings of $S^{4 n+3} / Q_{k}$. Let $M \nsubseteq R^{m}$ (or $M \not \subset R^{m}$ ) denote nonexistence of a $C^{\infty}$-immersion (or a $C^{\infty}$-embedding) of $M$ in $R^{m}$. Let $\nu(n)$ be the nonnegative integer such that $n=q \cdot 2^{\nu(n)}$, where $q$ is odd. Our main theorem is

THEOREM 1.1. If $\nu\left({ }^{2 n+1+i}\right)<2 n+k-2 i+\varepsilon$, then $S^{4 n+3} / Q_{k} \not R^{4 n+2+2 i}$ and $S^{4 n+3} / Q_{k} \not \subset R^{4 n+3+2 i}$, where $\varepsilon=0$ if $n$ is even $>0$, and $\varepsilon=1$ if $n$ is odd.

Define

$$
N(n, k)=\max \left[i: 1 \leqslant i \leqslant n, \nu\left(\begin{array}{c}
2 n+1+i \\
i
\end{array}\right)<2 n+k-2 i+\varepsilon\right] .
$$

The case $N(n, k)=n$ was obtained by Pitt [8, Corollary 5.6], and the case $k=3$ was obtained by K. Fujii. It follows from Theorem 1.1, for example, that

$$
\begin{aligned}
& S^{15} / Q_{k} \not R^{20}, \quad S^{15} / Q_{k} \not \subset R^{21} ; \quad S^{31} / Q_{3} \not R^{42}, \quad S^{31} / Q_{3} \not \subset R^{43}, \\
& S^{31} / Q_{k} \not \subset R^{44}, \quad S^{31} / Q_{k} \not \subset R^{45} \quad \text { for } k \geqslant 4 \text {. }
\end{aligned}
$$

Received by the editors August 19, 1982 and, in revised form, November 29, 1982.

1980 Mathematics Subject Classification. Primary 57R42; Secondary 57R40, 55N15, 55S25.

Key words and phrases. Quaternionic spherical space forms, representation ring, $K$-theory, $K O$-theory, $\gamma$-operations, immersions, embeddings. 
The complex representation ring $R_{C}\left(Q_{k}\right)$ of $Q_{k}$ is generated as a free abelian group by $1, a, b, c$ and $d_{r}\left(r=1,2, \ldots, 2^{k-2}-1\right)$ defined below (cf. $[2, \S 47.15 ; \mathbf{8}, \S 1$ and $3, \S 3])$ :

$$
\begin{aligned}
\left\{\begin{array} { l } 
{ 1 ( x ) = 1 , } \\
{ 1 ( y ) = 1 , }
\end{array} \quad \left\{\begin{array}{l}
a(x)=1, \\
a(y)=-1,
\end{array}\right.\right. & \left\{\begin{array} { l } 
{ b ( x ) = - 1 , } \\
{ b ( y ) = 1 , }
\end{array} \quad \left\{\begin{array}{l}
c(x)=-1, \\
c(y)=-1,
\end{array}\right.\right. \\
d_{r}(x)=\left[\begin{array}{ll}
\omega^{r} & 0 \\
0 & \omega^{-r}
\end{array}\right], & d_{r}(y)=\left[\begin{array}{cc}
0 & (-1)^{r} \\
1 & 0
\end{array}\right],
\end{aligned}
$$

where $\omega$ is a primitive $2^{k-1}$ th $\operatorname{root} \exp \left(2 \pi i / 2^{k-1}\right)$ of unity. The multiplicative structure is given by

$$
a^{2}=b^{2}=c^{2}=1, \quad a b=c, \quad d_{r} d_{s}=d_{r+s}+d_{r-s}, \quad b d_{r}=d_{2^{k-2}-r},
$$

where we define

$$
d_{0}=1+a, \quad d_{2^{k-2}}=b+c, \quad d_{-r}=d_{r}, \quad d_{2^{k-2}+r}=d_{2^{k-2}-r} .
$$

The reduced representation ring $\tilde{R}_{C}\left(Q_{k}\right)$ is generated as a free abelian group by $\alpha=a-1, \beta=b-1, \gamma=a+b+c-3, \delta_{r}=d_{r}-2\left(r=1,2, \ldots, 2^{k-2}-1\right)$ with relations (cf. [3, Proposition 3.3]):

$$
\begin{aligned}
& \alpha^{2}=-2 \alpha, \quad \beta^{2}=-2 \beta, \quad \gamma=\alpha \beta+2 \alpha+2 \beta, \quad \alpha \delta_{1}=-2 \alpha, \\
& \beta \delta_{1}=-2 \beta+\delta_{2^{k-2}-1}-\delta_{1}, \quad \delta_{r+1}=\delta_{1} \delta_{r}+2 \delta_{1}+2 \delta_{r}-\delta_{r-1}
\end{aligned}
$$

where $\delta_{2^{k-2}}=\gamma-\alpha, \delta_{0}=\alpha$. Thus $\tilde{R}_{C}\left(Q_{k}\right)$ is generated by $\alpha, \beta$ and $\delta_{1}$ as a ring.

Let $c_{R}: R_{R}\left(Q_{k}\right) \rightarrow R_{C}\left(Q_{k}\right)$ be the complexification. The real representation ring $R_{R}\left(Q_{k}\right)$, considered as the subring $c_{R}\left(R_{R}\left(Q_{k}\right)\right)$ of $R_{C}\left(Q_{k}\right)$, is generated by $1, a, b, c, d_{2 r}$ and $2 d_{2 r+1}\left(r=0,1, \ldots, 2^{k-3}-1\right)$ (cf. [8, Proposition 1.5]).

Define elements $v$ and $z$ in $\tilde{R}_{R}\left(Q_{k}\right)$ by

$$
c_{R}^{-1}\left(2 \delta_{1}\right)=v, \quad c_{R}^{-1}\left(\delta_{1}^{2}\right)=z .
$$

Let $\lambda$ be the canonical complex plane bundle over the quaternion projective space $H P^{n}=S^{4 n+3} / S^{3}$, and let $\pi: S^{4 n+3} / Q_{k} \rightarrow H P^{n}$ be the natural projection. Let $\xi_{C}$ : $\tilde{R}_{C}\left(Q_{k}\right) \rightarrow \tilde{K}\left(S^{4 n+3} / Q_{k}\right)$ be the projection defined in $[3, \S 4]$ and put $\delta=\xi_{C}\left(\delta_{1}\right)$. Then we have $\delta=\pi^{*} \lambda-2$ (cf. [3, Lemma 4.4]). The order $\# \delta^{i}$ of $\delta^{i} \in \tilde{K}\left(S^{4 n+3} / Q_{k}\right)$ is determined by H. Oshima in [7, Proposition 5.2] and T. Mormann in [6, Chapter 2, Theorem 4.52] as follows.

Proposition 1.3. $\# \delta^{i}=2^{2 n+k-2 i}(1 \leqslant i \leqslant n)$.

Let $r_{C}: \tilde{K}(X) \rightarrow \widetilde{K O}(X)$ and $c_{R}: \widetilde{K O}(X) \rightarrow \tilde{K}(X)$ be the realification and the complexification, respectively. Let $\xi_{R}: \tilde{R}_{R}\left(Q_{K}\right) \rightarrow \widetilde{K O}\left(S^{4 n+3} / Q_{k}\right)$ be the projection defined in $[4,(3.9)]$ (or in $[8$, Theorem 2.5]). Then, by (1.2),

$$
\xi_{R} v=r_{C}\left(\pi^{*} \lambda-2\right) \text { and } \xi_{R} z=c_{R}^{-1}\left(\left(\pi^{*} \lambda-2\right)^{2}\right)
$$

(cf. [4, Lemma 3.10]), because $\delta_{1}$ is self-conjugate and $c_{R} r_{C}=1+$ conjugation. For simplicity we write $v$ and $z$ instead of $\xi_{R} v$ and $\xi_{R} z$. Then, for the complexification $c_{R}: \widetilde{K O}\left(S^{4 n+3} / Q_{k}\right) \rightarrow \tilde{K}\left(S^{4 n+3} / Q_{k}\right)$, we have

$$
c_{R}(v)=2 \delta, \quad c_{R}(z)=\delta^{2} .
$$


Let $\# a$ (or $\# A$ ) denote the order of an element $a$ (or a group $A$ ). The orders of the canonical elements in $\widetilde{K O}\left(S^{4 n+3} / Q_{k}\right)$ are determined as follows.

THEOREM 1.5. For $z^{i}$ and $v z^{i} \in \widetilde{K O}\left(S^{8 m+7} / Q_{k}\right)$,

$$
\begin{aligned}
\# z^{i} & =2^{4 m+k-4 i+3} & & (0<i \leqslant m), \\
\# v z^{i} & =2^{4 m+k-4 i} & & (0 \leqslant i \leqslant m) .
\end{aligned}
$$

THEOREM 1.6. For $z^{i}$ and $v z^{i} \in \widetilde{K O}\left(S^{8 m+3} / Q_{k}\right)$,

$$
\begin{aligned}
\# z^{i} & =2^{4 m+k-4 i} & & (0<i \leqslant m), \\
\# v z^{i} & =2^{4 m+k-4 i-3} & & (0 \leqslant i<m) .
\end{aligned}
$$

Corollary 1.7. For $v \in \widetilde{K O}\left(S^{4 n+3} / Q_{k}\right)$,

$$
\# v= \begin{cases}2^{2 n+k-2} & \text { if } n \text { is odd } \\ 2^{2 n+k-3} & \text { if } n \text { is even }>0 .\end{cases}
$$

K. Fujii [4] proved the result for $k=3$. H. Öshima [7] announced it for $k=4$ and conjectured Corollary 1.7 .

The author wishes to express his sincere thanks to the referee for his valuable suggestions.

2. Proofs of Theorems 1.5 and 1.6. First we prepare a lemma.

LEMMA 2.1. In $\widetilde{K O}\left(S^{4 n+3} / Q_{k}\right)$ :

$$
\begin{gathered}
2^{k} v+\sum_{j=1}^{2^{k-3}}\left(2 c_{2 j}+c_{2 j+1} v\right) z^{j}=0, \\
2^{k} z+\sum_{j=1}^{2^{k-3}}\left(2^{-1} c_{2 j} v+c_{2 j+1} z\right) z^{j}=0,
\end{gathered}
$$

where $c_{s}$ are integers satisfying

$$
\begin{aligned}
c_{2} & =2^{k-2}\left(2^{2 k-3}+1\right) / 3, \quad c_{2^{k-2}+1}=1, \\
\nu\left(c_{s}\right) & \geqslant \max (1, k-s) \quad \text { for } 0<s \leqslant 2^{k-2} .
\end{aligned}
$$

Proof. It was proved in [6] that $\sum_{s=1}^{2^{k-2}+1} c_{s} \delta_{1}^{s}=0$ in $\tilde{R}_{C}\left(Q_{k}\right)$, where

$$
c_{s}=\left(\begin{array}{c}
2^{k-2}+s \\
2 s-1
\end{array}\right)+2\left(\begin{array}{c}
2^{k-2}+s-1 \\
2 s-1
\end{array}\right)+\left(\begin{array}{c}
2^{k-2}+s-2 \\
2 s-1
\end{array}\right) .
$$

(*) follows easily from

$$
\nu\left(\begin{array}{c}
m \\
n
\end{array}\right)=\alpha(n)+\alpha(m-n)-\alpha(m),
$$

where $\alpha(n)$ denotes the number of nonzero terms in the dyadic expansion of $n$. (a) and (b) are proved by multiplying this by 2 and $\delta_{1}$, respectively, and applying $c_{R}^{-1} \xi_{C}$. 
LEMMA 2.2. In $\widetilde{K O}\left(S^{8 m+7} / Q_{k}\right)$ :

$$
\begin{aligned}
z^{m+1} & =0, & & \\
2^{4 i+k} v z^{m-i} & =0 & & (0 \leqslant i \leqslant m), \\
2^{4 i+k+3} z^{m-i} & =0 & & (0 \leqslant i<m), \\
2^{4 i+k-1} v z^{m-i}+2^{4 i+k+2} z^{m-i} & =0 & & (0 \leqslant i<m), \\
2^{4 i+k+2} z^{m-i}+2^{4 i+k+3} v z^{m-i-1} & =0 & & (0 \leqslant i<m) .
\end{aligned}
$$

Proof. (i) follows from [8, Theorem 2.5].

(ii) and (iii) are proved by induction on $i$. (ii) for $i=0$ follows from (i) and (a) $\times z^{m}$. (iii) for $i=0$ follows from (b) $\times 2^{3} z^{m-1}$ and (ii) for $i=0$. (iii) for any $j \leqslant i-1$, (ii) for any $j \leqslant i$ and (b) $\times 2^{4 i+3} z^{m-i-1}$ imply (iii) for $j=i$. (ii) for any $j \leqslant i$, (iii) for any $j \leqslant i$ and (a) $\times 2^{4 i+4} z^{m-i-1}$ imply (ii) for $j=i+1$.

Using (b) $\times 2^{4 i+2} z^{m-i-1}$ (resp. (a) $\times 2^{4 i+3} z^{m-i-1}$ ) and (i) (iii), we obtain (iv) (resp. (v)).

\section{LEMMA 2.3. In $\widetilde{K O}\left(S^{8 m+3} / Q_{k}\right)$ :}

(i)

$$
\begin{aligned}
z^{m+1} & =0, & & \\
v z^{m} & =0, & & \\
2^{4 i+k} z^{m-i} & =0 & & (0 \leqslant i<m), \\
2^{4 i+k+1} v z^{m-i-1} & =0 & & (0 \leqslant i<m), \\
2^{4 i+k-1} z^{m-i}+2^{4 i+k} v z^{m-i-1} & =0 & & (0 \leqslant i<m), \\
2^{4 i+k} v z^{m-i-1}+2^{4 i+k+3} z^{m-i-1} & =0 & & (0 \leqslant i<m-1) .
\end{aligned}
$$

Proof. (i) follows from Lemma 2.2(i) and the naturality. (ii) is proved in $[4, \S 4]$. The proofs of (iii) $\sim$ (vi) are similar to those of Lemma 2.2(ii) $\sim(v)$, so we omit the details.

Proof of Theorem 1.5. By Lemma 2.2(iii), (ii) we have

$$
\# z^{i} \leqslant 2^{4 m+k-4 i+3} \quad(0<i \leqslant m) \quad \text { and } \quad \# v z^{i} \leqslant 2^{4 m+k-4 i} \quad(0 \leqslant i \leqslant m) .
$$

Let $j: S^{8 m+3} / Q_{k} \rightarrow S^{8 m+7} / Q_{k}$ be the natural inclusion. Then it follows from $[4, \S 4]$ that $\operatorname{Ker} j^{*}$, the kernel of the induced homomorphism $j^{*}: \widetilde{K O}\left(S^{8 m+7} / Q_{k}\right) \rightarrow$ $\widetilde{K O}\left(S^{8 m+3} / Q_{k}\right)$, is generated by $v z^{m}$. According to [5, Chapter 6, Proposition 5.7],

$$
\# \widetilde{K O}\left(S^{8 m+7} / Q_{k}\right)=2^{2 m k+4 m+k+4} \text { and } \# \widetilde{K O}\left(S^{8 m+3} / Q_{k}\right)=2^{2 m k+4 m+4} \text {. }
$$

Hence, by Lemma 2.2(ii), we obtain

$$
2^{k}=\# \widetilde{K O}\left(S^{8 m+7} / Q_{k}\right) / \# \widetilde{K O}\left(S^{8 m+3} / Q_{k}\right) \leqslant \# \operatorname{Ker} j^{*}=\# v z^{m} \leqslant 2^{k}
$$

Thus \#vz $z^{m}=2^{k}$. Therefore, by Lemma 2.2(iv), (v) we have

$$
2^{4 m+k-4 i+2} z^{i}=-2^{4 m+k-4 i-1} v z^{i}=\cdots=-2^{k-i} v z^{m} \neq 0 .
$$

Proof of Theorem 1.6. By Lemma 2.3(iii), (iv) we have

$$
\# z^{i} \leqslant 2^{4 m+k-4 i} \quad(0<i \leqslant m) \quad \text { and } \quad \# v z^{i} \leqslant 2^{4 m+k-4 i-3} \quad(0 \leqslant i<m) .
$$


By Lemma 2.3(vi), (v) we have

$$
2^{4 m+k-4 i-1} z^{i}=-2^{4 m+k-4 i-4} v z^{i}=\cdots=-2^{4 m+k-4} v .
$$

But, by (1.4) and Proposition 1.3, $c_{R}\left(2^{4 m+k-4} v\right)=2^{4 m+k-3} \delta \neq 0$, so $2^{4 m+k-4} v \neq 0$.

3. Atiyah's criterion. Exterior power operation $\lambda^{i}(\alpha), \alpha \in R_{C}\left(Q_{k}\right)$, is determined by

$$
\begin{gathered}
\lambda^{0}(\alpha)=1, \quad \lambda^{1}(\alpha)=\alpha, \quad \lambda^{i}(\alpha)=0 \text { for } i>1, \alpha=1, a, b, c, \\
\lambda^{2}\left(d_{r}\right)=1 \text { for } r \text { odd, } \quad \lambda^{2}\left(d_{r}\right)=a \text { for } r \text { even, } \lambda^{i}\left(d_{r}\right)=0 \text { for } i>2 .
\end{gathered}
$$

Define $\lambda_{t}(\alpha)=\sum_{i \geqslant 0} \lambda^{i}(\alpha) t^{i}$. Then the Grothendieck $\gamma$-operations $\gamma^{i}$ are obtained from the equality of the polynomials

$$
\lambda_{t /(1-t)}(\alpha)=\gamma_{t}(\alpha)=\sum_{i \geqslant 0} \gamma^{i}(\alpha) t^{i}
$$

The following is well known (cf. [8, p. 2]).

LeMma 3.1. $\gamma_{t}\left(\delta_{2 r+1}\right)=1+\delta_{2 r+1}\left(t-t^{2}\right)$, where $\delta_{2 r+1}=d_{2 r+1}-2 \in \tilde{R}_{C}\left(Q_{k}\right)$.

Let $v$ and $z$ be the elements in $\tilde{R}_{R}\left(Q_{k}\right)$ defined in (1.2). Then we prove

LEMMA 3.2. $\gamma_{t}(v)=1+v\left(t-t^{2}\right)+z\left(t-t^{2}\right)^{2}$.

Proof. Since $\gamma_{t}$ is natural with respect to the complexification $c_{R}$, we have, by Lemma 3.1 and (1.2),

$$
\begin{aligned}
\gamma_{t}(v) & =\gamma_{t} c_{R}^{-1}\left(2 \delta_{1}\right)=c_{R}^{-1}\left\{\gamma_{t}\left(\delta_{1}\right)\right\}^{2}=c_{R}^{-1}\left\{1+\delta_{1}\left(t-t^{2}\right)\right\}^{2} \\
& =c_{R}^{-1}\left\{1+2 \delta_{1}\left(t-t^{2}\right)+\delta_{1}^{2}\left(t-t^{2}\right)^{2}\right\}=1+v\left(t-t^{2}\right)+z\left(t-t^{2}\right)^{2} .
\end{aligned}
$$

As an application of Grothendieck $\gamma$-operations in $\mathrm{KO}$-theory, $\mathrm{M}$. F. Atiyah [1] obtained the following

THEOREM 3.3. Let $M$ be an $n$-dimensional compact smooth manifold and $\tau_{0} \in \overparen{K O}(M)$ the stable class of the tangent bundle of $M$. Then, if $M$ is immersible (resp. embeddable $)$ in $R^{n+r}, \gamma^{i}\left(-\tau_{0}\right)=0$ for all $i>r($ resp. $i \geqslant r)$.

LEMMA 3.4. Let $\tau_{0}$ be the stable class of the tangent bundle $\tau=\tau\left(S^{4 n+3} / Q_{k}\right)$ of $S^{4 n+3} / Q_{k}$. Then

$$
\gamma_{t}\left(-\tau_{0}\right)=\sum_{i \geqslant 0}\left(\begin{array}{c}
2 n+1+2 i \\
2 i
\end{array}\right) z^{i}\left(t-t^{2}\right)^{2 i}-\sum_{i \geqslant 0} \frac{1}{2}\left(\begin{array}{c}
2 n+2+2 i \\
2 i+1
\end{array}\right) v z^{i}\left(t-t^{2}\right)^{2 i+1}
$$

Proof. According to [9, Corollary 3.3],

$$
-\tau_{0}=4 n+3-\tau=4(n+1)-(n+1)\left(r_{C} \pi^{*} \lambda\right)=-(n+1) v .
$$


By Lemma 3.2 and (1.2), we have

$$
\begin{aligned}
& \gamma_{t}\left(-\tau_{0}\right)=\gamma_{t}(-(n+1) v)=\left(\gamma_{t}(v)\right)^{-n-1}=\left\{1+v\left(t-t^{2}\right)+z\left(t-t^{2}\right)^{2}\right\}^{-n-1} \\
& =c_{R}^{-1}\left\{1+\delta_{1}\left(t-t^{2}\right)\right\}^{-2 n-2} \\
& =c_{R}^{-1}\left\{\sum_{j \geqslant 0}(-1)^{j}\left(\begin{array}{c}
2 n+1+j \\
j
\end{array}\right) \delta_{1}^{j}\left(t-t^{2}\right)^{j}\right\} \\
& =c_{R}^{-1}\left\{\sum_{i \geqslant 0}\left(\begin{array}{c}
2 n+1+2 i \\
2 i
\end{array}\right) \delta_{1}^{2 i}\left(t-t^{2}\right)^{2 i}\right. \\
& \left.-\sum_{i \geqslant 0} \frac{1}{2}\left(\begin{array}{c}
2 n+2+2 i \\
2 i+1
\end{array}\right) 2 \delta_{1}^{2 i+1}\left(t-t^{2}\right)^{2 i+1}\right\} \\
& =\sum_{i \geqslant 0}\left(\begin{array}{c}
2 n+1+2 i \\
2 i
\end{array}\right) z^{i}\left(t-t^{2}\right)^{2 i}-\sum_{i \geqslant 0} \frac{1}{2}\left(\begin{array}{c}
2 n+2+2 i \\
2 i+1
\end{array}\right) v z^{i}\left(t-t^{2}\right)^{2 i+1} .
\end{aligned}
$$

Now we are ready to prove Theorem 1.1.

Proof of Theorem 1.1. Let $n$ be even. Let $y_{i}=z^{i / 2}$ if $i$ is even and $y_{i}=$ $-2^{-1} v z^{(i-1) / 2}$ if $i$ is odd. Then, by Theorem 1.6, $\# y_{i}=2^{2 n+k-2 i}$ for $i \leqslant n$ and $y_{i}=0$ for $i>n$. Also, by Lemma 3.4,

$$
\begin{aligned}
\gamma_{t}\left(-\tau_{0}\right) & =\sum_{l}\left(\begin{array}{c}
2 n+1+l \\
l
\end{array}\right) y_{l} t^{\prime}(1-t)^{l} \\
& =\sum_{s}(-1)^{s} t^{s} \sum_{2^{-1} s \leqslant l \leqslant s}(-1)^{l}\left(\begin{array}{c}
l \\
s-l
\end{array}\right)\left(\begin{array}{c}
2 n+1+l \\
l
\end{array}\right) y_{l} .
\end{aligned}
$$

Hence, if $S^{4 n+3} / Q_{k}$ is immersed in $R^{4 n+2+2 i}$, then for all $s \geqslant 2 i$,

$$
\sum_{2^{-1} s \leqslant l \leqslant s}(-1)^{l}\left(\begin{array}{c}
l \\
s-l
\end{array}\right)\left(\begin{array}{c}
2 n+1+l \\
l
\end{array}\right) y_{l}=0 .
$$

The desired equalities

$$
\left(\begin{array}{c}
2 n+1+l \\
l
\end{array}\right) y_{l}=0
$$

are obtained by a downward induction on $s$, beginning with $s=2 n$.

The other cases are similar.

\section{REFERENCES}

1. M. F. Atiyah, Immersions and embeddings of manifolds, Topology 1 (1962), 125-132.

2. C. W. Curtis and I. Reiner, Representation theory of finite groups and associative algebras, Wiley, New York, 1962.

3. K. Fujii, On the $K$-ring of $S^{4 n+3} / H_{m}$, Hiroshima Math. J. 3 (1973), 251-265.

4. On the KO-ring of $S^{4 n+3} / H_{m}$, Hiroshima Math. J. 4 (1974), 459-475.

5. N. Mahammed, R. Piccinini and U. Suter, Some applications of topological K-theory, North-Holland Math. Studies no. 45, North-Holland, Amsterdam, 1980.

6. T. Mormann, Topologie Sphärischer Raumformen, Dissertation, Univ. Dortmund, 1978.

7. H. Ōshima, On stable homotopy types of some stunted spaces, Publ. Res. Inst. Math. Sci. 11 (1976), 497-521.

8. D. Pitt, Free actions of generalized quaternion groups on spheres, Proc. London Math. Soc. (3) 26 (1973), 1-18.

9. R. H. Szczarba, On tangent bundles of fibre spaces and quotient spaces, Amer. J. Math. 86 (1964), 685-697.

Department of Mathematics, Faculty of Science, Kochi University, Kochi 780, JaPan 